\title{
Combined n-3 Fatty Acids and $\alpha$-Tocopherol Supplementation Improved the Ovine Sperm Cryosurvival
}

\author{
Armin Towhidi ${ }^{1,{ }^{*}}$, Saeed Zeinoaldini ${ }^{1}$, Rouzbeh Ardebili ${ }^{1}$, Navid Dadashpour Davachi ${ }^{1}$, \\ Amir Hossein Nasiri ${ }^{1}$ \\ ${ }^{1}$ Department of Animal Science, Faculty of Agricultural Science and Engineering, University of Tehran, Karaj, IR Iran \\ ${ }^{*}$ Corresponding author: Armin Towhidi, Department of Animal Science, Faculty of Agricultural Science and Engineering, University of Tehran, Karaj, IR Iran, Tel: + 98-9121404081, Fax: \\ +98-2632246752, E-mail: atowhidi@ut.ac.ir
}

Received: August 27, 2013; Revised: September 29, 2013; Accepted: September 30, 2013

\begin{abstract}
Background: Spermatozoa preservation is an approach to improve the fertility rate to pass on the valuable genetic materia from sire to their offspring. During the last 20 years, reproductive biotechnologists have focused on the approaches that improve spermatozoa cryosurvival. One of the possible mechanisms is supplementation in semen extender.

Objectives: The aim of this study was to evaluate the combined effects of healthy ram semen with n-3 fatty acids and $\alpha$-tocopherol (Vitamin E) on freezing ability and fatty acid (FA) content of sperm cell.

Materials and Methods: Semen collection was performed on six mature Zandi rams by an artificial vagina. In the present study, two experiments were carried out. In Experiment 1, the specimen quality was assessed. Then the samples were pooled. The pooled

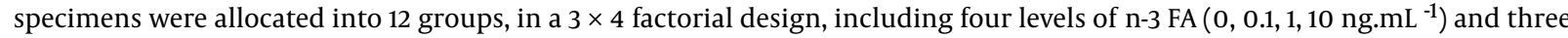
levels of $\alpha$-tocopherol $(0.0 .1,0.2 \mathrm{mM})$. Then sperm critical characteristics such as proportion of motile sperm, progressive motile sperm, viable and abnormal sperms were measured. Furthermore, after freezing-thawing procedure, the recovery rate was considered as a vital indicator of semen quality. After thawing, the highest progressive motility was obtained when treated with $0.1 \mathrm{mM} \alpha$-tocopherol and $1 \mathrm{ng} \cdot \mathrm{mL}^{-1} \mathrm{n}-3 \mathrm{FA}$. So, the second experiment was designed to measure the content of FA in specimens that fortified with $0.1 \mathrm{mM} \alpha$-tocopherol, 1 ng.mL ${ }^{-1} \mathrm{n}-3 \mathrm{FA}$ and also in groups without $\alpha$-tocopherol and FA.

Results: The data showed that before freezing, docosahexaenoic acid (DHA) level of sperm was increased when the FA introduced into extender $(\mathrm{P} \leq \mathrm{0.01})$. On the other hand, in the FA group, the n-3 FA and polyunsaturated fatty acid content were significantly higher compared with n-6 FA and saturated fatty acid level. However, in other groups, there were no significant alteration in the overall proportion of $n-3$ FA and $n-6$ FA were recorded $(P \leq 0.01)$.

conclusions: It was concluded that the cryosurvival of ram semen could be improved by adding DHA along with alfa-tocopherol as an antioxidant.
\end{abstract}

Keywords: $\alpha$-tocopherol; Freezing; n-3 Fatty Acids; Sheep; Sperm

\section{Background}

Cryopreservation is known as a classical semen storage technique which has a number of advantages; however, thawing and freezing induce some detrimental effects on sperm ultra-structure and biochemical and functional structures (1), leading to the reduction of motility, membrane integrity and fertilizing potential (2).

The main purpose of freezing extender is to provide an energy source for spermatozoa to protect them from damages caused by temperature fluctuations and maintain a suitable temporary environment to survive. At present, the egg yolk is a common component of semencryopreservation extenders of domestic animals. It has been shown that egg yolk has some advantageous effects such as protecting the plasma membrane of sperm cryo- preservation against temperature-related injuries, in association with other components (3). It is reported that egg yolk has a high risk of contamination (4), commercial extenders with an egg yolk substitute have been recently available for freezing animal semen $(5,6)$. However, little information is known about ram sperm preservation.

The sperm plasma membrane is a highly dynamic construction that can regulates the extracellular exchanges and induces the process of fertilization (7). The difference in lipid composition of the sperm plasma membrane is a key factor in the capability of sperm freezing (8). In various mammalian species, up to $60 \%$ of the total fatty acids consisted of long-chain polyunsaturated fatty acids (LCPUFA) of the n-3 series (9). This specific lipid composition causes fluidity and flexibility of the cell membrane which are resulted from the presence of the several double 
bond fatty acids. This specific physical changes in membrane may provide more resistance against damages arising from icy crystal formation (10). It has been suggested that High DHA concentration in both semen and sperm is positively associated with sperm motility in humans ( 9 , 11). The membrane fluidity is associated to the availability of docosahexaenoic acid which is essential for the motility of sperm tails (12). It has been observed that the sperm obtained from as the nozoospermic men had lower levels of DHA compared with sperm from normozoospermic men (9). The reduction of semen Polly unsaturated fatty acid (PUFA) concentration decreases the sperm cell number, motility and sperm fertilizing potential in animals (13). Due to high level of phospholipid and unsaturated fatty acid content of the mammalian sperm membrane, these cells are sensitive to lipid peroxidation, (14). The Seminal plasma protects the cells against this process by antioxidants. Moreover, diluting semen during cryopreservation decreases the availability of plasma semen PUFA and antioxidant of sperm cell.

\section{Objectives}

Therefore, the objective of the present study was to evaluate the effects of adding different levels of n-3 fatty acid and alfa-tocopherol to an egg-yolk-free extender in order to assess the freezing ability of ram sperm.

\section{Materials and Methods}

\subsection{Animal and Location}

Six mature Zandi rams with average weight of $61-68 \mathrm{~kg}$ (2 - 3 years old) from the sheep flock of the Department of Animal Science, University of Tehran, located in Karaj $\left(35^{\circ} 48^{-} \mathrm{N}, 51^{\circ} 2^{-} \mathrm{E}\right)$ were subjected to the present study in autumn 2011.

Experimental procedures were approved by the University of Tehran Animal Welfare and Ethics Committee.

\subsection{Experimental Design}

In Experiment 1, semen samples collected by a ram artificial vagina, twice a week for four weeks (totally 48 ejaculations). After semen collection, the semen specimens were transferred to the laboratory of the Animal Breeding Center of Iran, and reserve at $34^{\circ} \mathrm{C}$ water bath. Specimens were subjected to further analysis using a phase-contrast microscope at 400X. The specimens with $\geq 70 \%$ motility were selected. Then the selected samples were pooled and extended using an Andromed extender (Minitüb, Tiefenbach, Germany). The Andromed extender was supplemented with different levels of n-3 FA (0, 0.1, 1 and 10 ng.mL ${ }^{-1}$; Viva Pharmaceutical Inc, Canada;) and $\alpha$-tocopherol (0, 0.1, 0.2 mM; Sigma Chemical Co., St. Louis, MO, USA). To obtain different concentration of FA and $\alpha$-tocopherol; the n-3 FA and $\alpha$-tocopherol were solved in pre warmed $\left(37^{\circ} \mathrm{C}\right)$ test tubes containing Ethanol. Afterward, to prepare the tubes in a way that they possess a thin layer of FA and $\alpha$-tocopherol on their inner surface, ethanol was allowed to evaporate (15). Then the extended semen was added to the tubes and incubated for 20 minutes at $37^{\circ} \mathrm{C}$ to allow the spermatozoa to uptake fatty acid and alfa-tocopherol (16). Five samples of fresh and frozen-thawed semen from each treatment were pooled for semen quality evaluation. Eight replicates were used for each treatment.

In Experiment 2, the $\alpha$-tocopherol plus FA group and the control group were considered as the core of study. In this part of study, collected semen from rams was divided into two groups (with or without FA and $\alpha$-tocopherol). Then the groups were frozen for further studies. The content of sperm fatty acid profile was identified before freezing and after thawing.

\subsection{Freezing and Thawing Process}

At the first step, semen was diluted. Then the diluted semen was cooled at $4-5{ }^{\circ} \mathrm{C}$ for 2 hours. Following the cooling process, semen was frozen in straws. For semen cryopreservation, samples were stored in a Styrofoam box containing liquid nitrogen. Racks that contain the samples were placed into the liquid nitrogen vapor at a height of $4 \mathrm{~cm}$ above the liquid for 8 minutes, and then the straws were plunged into liquid nitrogen. For thawing process, the straws were placed into a water bath adjusted at $37^{\circ} \mathrm{C}$ for 30 seconds (16).

\subsection{Semen Evaluation}

\subsubsection{Post-Thawed Sperm Motility and Progressive Mo- tility}

Pre-warmed slide and cover slip were used to evaluate sperm motility (without CASA program). At first step, a drop of thawed semen loaded on a pre-warmed slide. Then it was covered by a cover slip. The percentage of motile sperms and sperms with progressive motility were evaluated under a phase-contrast microscope at 400X magnification. The mean of five observations was considered as a single data point.

\subsubsection{Sperm Viability}

A small drop of fresh or frozen-thawed semen was loaded on a pre-warmed slide and mixed with a relatively larger drop of the supravital stain $[1 \%(\mathrm{w} / \mathrm{v})$ Eosin B, $5 \%(\mathrm{w} / \mathrm{v})$ nigrosin in $3 \%$ tri-sodium citrate dihydrate solution] to prepare a thin and uniform smear (15). Following air-drying, the smear was evaluated using a phase-contrast microscope (400X). Ten scopes (thirty three spermatozoa per scope) that contained three hundred and thirty three spermatozoa were counted. Spermatozoa with unstained heads considered as live sperms and spermatozoa with stained or partial 
Towhidi A et al.

stained heads counted as dead sperms (16).

\subsubsection{Sperm Abnormality}

For the evaluation of total abnormalities in the semen samples, at least three drops of the semen were pipetted into $1.5 \mathrm{~mL}$ tubes, containing $1 \mathrm{~mL}$ Hancock's solution. One drop of this mixture was placed on a microscope slide and covered with a cover slip.

\subsubsection{Recovery Rate (RR)}

This equation; $\mathrm{RR}=$ Motility after freezing / Motility before freezing, was used for calculation of the spermatozoa recovery rate.

\subsection{Fatty Acid Composition of Sperm}

Using an equal volume of $\mathrm{NaCl}(0.85 \%, \mathrm{w} / \mathrm{v})$, collected semen from the treated and control group were diluted. Then for separation of seminal plasma from the cell pellet the diluted semen was centrifuged at $1000 \mathrm{~g}$ for 10 minutes at room temperature (17). The supernatant was transferred to a fresh test tube, and the cell pellet washed with $1 \mathrm{~mL}$ of $0.85 \%$ (wt/vol) $\mathrm{NaCl}$ and re-centrifuged using the same procedure as described by Sariozkan et al. (18). The sperm cell pellet was suspended and centrifuged in the presence of $2 \mathrm{~mL} \mathrm{NaCl}(0.85 \%, \mathrm{w} / \mathrm{v})$. Then obtained pellet was washed twice with saline solution (17). The lipid extraction was performed following application of a proper combination of chloroform - methanol $(2: 1, \mathrm{v} / \mathrm{v})$ to make a homogenize sperm (19). By using Metcalf method, the specimens were trans-methylated(20). Using a capillary column system, Carbowax, $30 \mathrm{~m} \times 0.25 \mathrm{~mm}$ in diameter, $0.25 \mu \mathrm{m}$ film thickness (Alltech Ltd., Carnforth, Lancashire, UK), the gas chromatography was conducted for analyzing the resultant fatty acid methyl esters (HP6890 with FID detector and auto-sampler HP7683, Hewlett Packard, Wilmington, DE, USA) (4). Integration of the peaks and subsequent data handling was performed using the HP Chemstation software (Hewlett Packard), enabling determination of the fatty acid composition (proportion of total fatty acids) by comparison of the total fatty acid peak areas to that of the pentadecanoic fatty acid standard. The identities of the peaks were verified by comparison with the retention times of standard fatty acid methyl esters.

\subsection{Statistical Analysis}

Data were analyzed using GLM procedure of SAS 9.1 in experiment 1 and 2 (SAS Institute, Cary, NC, USA). Treatment mean comparisons were calculated using Duncan's multiple range test. The results were expressed as mean \pm
SEM. Differences with values of $\mathrm{P}<0.05$ were considered statistically significant.

\section{Results}

\subsection{Experiment 1}

The mean percentages of post-thawed sperm in different groups are shown in Table 1. Following thawing, the treated group with $0.1 \mathrm{mmol}$ alfa-tocopherol and $1 \mathrm{ng} . \mathrm{mL}$ ${ }^{-1} \mathrm{n}-3$ FA ( $\alpha$-tocopherol plus FA group) showed better vital features compared with other groups (control and treatment groups). The sperm motility was not affected by n-3 FA and $\alpha$-tocopherol. The percentage of sperm motility $(\mathrm{MOT})($ mean \pm SEM $)$ was $61.43 \pm 0.59,61.63 \pm 0.59$ and 61.47 $\% \pm 0.59$ respectively for $0,0.1$ and $0.2 \mathrm{mM}$ alfa-tocopherol. Treated group with $0,0.1,1$ and $10 \mathrm{ng} \cdot \mathrm{mL}^{-1} \mathrm{n}-3$ FA showed the MOT percentages as follows; $61.95 \pm 0.70,61.12 \pm 0.70$, $61.93 \pm 0.70$ and $61.40 \pm 0.70$ respectively.

Sperm PMOT percentage was significantly affected by n-3 FA, $\alpha$-tocopherol and their interactions. The mean PMOT $( \pm$ SEM) percentage was $51.16 \pm 0.67,52.21 \pm 0.67$ and $51.70 \pm 0.67$ respectively for, $0,0.1,0.2 \mathrm{mM} \alpha$-tocopherol. Among three levels of $\alpha$-tocopherol, POMT in the treated group with $0 \mathrm{mM} \alpha$-tocopherol was significantly lower compared with other levels $(\mathrm{P} \leq 0.05)$. Treated group with 0.1 ng.mL ${ }^{-1} \mathrm{FA}(51.02 \pm 0.79)$ had lower PMOT percentage compared with groups with 0,1 and 10 ng.mL ${ }^{-1}$ FA. (51.95 $\pm 0.79,51.89 \pm 0.79$ and $52.01 \pm 0.79$, respectively). Among groups treated with FA, the groups with $1 \mathrm{ng} . \mathrm{mL}^{-1}$ n-3 FA and $0.1 \mathrm{mM}$ alfa-tocopherol showed higher values compared with other treated groups.

In the groups treated with various levels of alfa-tocopherol and FA, there were no significant differences in the percentage of viable sperm $(60.61 \pm 0.87,61.13 \pm 0.87$ and $60.86 \pm 0.87$ respectively for $0,0.1$ and $0.2 \mathrm{mM}$ alfatocopherol) and (60.92 $\pm 0.98,60.92 \pm 0.98,60.92 \pm 0.98$ and $60.92 \pm 0.98$ respectively for $0,0.1,1,10$ ng.mL ${ }^{-1} \mathrm{FA}$ ) (Table 1 ).

There was no significant difference in the percentage of abnormal sperm between groups at various levels of VE $(3.37 \pm 0.25,3.56 \pm 0.25$ and $3.45 \pm 0.25$ for $0,0.1,0.2 \mathrm{mM}$ levels of $\alpha$-tocopherol). Among various levels of FA, the lowest abnormality belonged to the group treated with $0.1 \mathrm{ng} . \mathrm{mL}^{-1}$ to find the detailed results refer to Table 1 .

Differences between recovery rates were not significant in groups at various levels of alfa-tocopherol and FA.

\subsection{Experiment 2}

Sperm membrane composition for fatty acids, n-3 and n-6 FA, MUFA percentages, n3 / n6 and PUFA / SFA ratio are shown in Table 2. Prior to freezing, DHA content was higher in FA group compared to groups without FA $(\mathrm{P}<0.05)$. There was a reduction in the ratio of n-3/n-6 FA following the thawing process in both groups (with and without 
Towhidi A et al.

FA). However, the proportion of n-3 / n-6 FA was higher in

FA group prior to the freezing procedure (Table 2).

Table 1. In vitro Characteristics of Post-Thawed Sperm at Different Levels of n-3 FA and alfa-Tocopherol in Ram

\begin{tabular}{|c|c|c|c|c|}
\hline Treatments & $\begin{array}{l}\text { Motility, }(\%)(\text { SEM }= \\
\text { 5.51) }\end{array}$ & $\begin{array}{l}\text { Progressive Motility, ( \%) (SEM } \\
=2.37 \text { ) }\end{array}$ & $\begin{array}{l}\text { Viability, }(\%)(\text { SEM }= \\
\text { 2.03) }\end{array}$ & $\begin{array}{l}\text { Abnormality, ( \%)(SEM } \\
=0.87 \text { ) }\end{array}$ \\
\hline FoV01(control) & 36.05 & $29.0^{\mathrm{bcd}}$ & 34.23 & 3.08 \\
\hline Fo.1Vo & 36.50 & $27.50^{d}$ & 35.05 & 3.69 \\
\hline FiVo & 36.50 & 29.50 bcd & 34.42 & 3.36 \\
\hline F10Vo & 36.0 & $29.70 \mathrm{bcd}$ & 34.84 & 3.23 \\
\hline FoVo.1 & 36.95 & $28.75^{\mathrm{cd}}$ & 35.14 & 3.60 \\
\hline Fo.1V0.1 & 36.85 & $30.50^{\mathrm{abc}}$ & 34.65 & 3.03 \\
\hline F1Vo.1 & 36.45 & $32.50^{a}$ & 34.94 & 3.42 \\
\hline F10Vo.1 & 36.70 & $29.50 \mathrm{bcd}$ & 35.57 & 3.38 \\
\hline FOVo.2 & 36.50 & $29.50 \mathrm{bcd}$ & 33.80 & 3.53 \\
\hline Fo / 1Vo.2 & 35.75 & $29.25^{\mathrm{bcd}}$ & 34.50 & 3.58 \\
\hline F1Vo.2 & 36.60 & $29.750^{b c}$ & 35.33 & 3.56 \\
\hline F10Vo.2 & 36.35 & $31.10^{\mathrm{ab}}$ & 34.46 & 3.92 \\
\hline
\end{tabular}

a,b,c, Values in each column that do not have any common letter are significantly ( $\mathrm{P} \leq 0.05)$ different.

Table 2. Fatty Acid Composition of Spermatozoa Lipid of Ram Semen in the Control Group (Without FA) and n-3 FA Group (With FA)

\begin{tabular}{|c|c|c|c|c|c|}
\hline \multirow[t]{2}{*}{ Fatty Acid } & \multicolumn{2}{|c|}{ Before Freezing } & \multicolumn{2}{|c|}{ After Thawing } & \multirow[t]{2}{*}{ SEM } \\
\hline & Without FA & With FA & Without FA & With FA & \\
\hline C14:0 & $5.06^{a b}$ & $6.91^{\mathrm{a}}$ & $1.63^{b}$ & $2.51 \mathrm{~b}$ & 3.81 \\
\hline C16:0 & $12.13^{\mathrm{C}}$ & $17.34^{\mathrm{a}}$ & $15.18^{b}$ & $14.77^{\mathrm{b}}$ & 8.11 \\
\hline C18:0 & $9.24^{\mathrm{a}}$ & $7.30^{b}$ & $7.24^{b}$ & $7.41^{b}$ & 2.40 \\
\hline C18:1 & $3.04^{\mathrm{ab}}$ & $3.51^{\mathrm{a}}$ & $1.85^{\mathrm{b}}$ & $1.94^{\mathrm{b}}$ & 1.35 \\
\hline C18:2 & $6.86^{\mathrm{a}}$ & $10.67^{b}$ & $6.61^{a}$ & $7.11^{\mathrm{a}}$ & 3.08 \\
\hline C18:3 & $0.37^{\mathrm{a}}$ & $0.89^{b}$ & $0.29^{a}$ & $0.13^{a}$ & 0.33 \\
\hline C20:4 & $0.65^{a b}$ & $1.19^{\mathrm{a}}$ & $0.15^{b}$ & $0.03^{b}$ & 0.67 \\
\hline EPA & 0.29 & 0.14 & Minor & Minor & 0.45 \\
\hline DHA & $5.54^{\mathrm{a}}$ & $11.92^{b}$ & $3.66^{c}$ & $2.12^{\mathrm{C}}$ & 3.62 \\
\hline n-3 & $6.18^{a}$ & $12.97^{\mathrm{b}}$ & $3.96^{\mathrm{c}}$ & $3.26^{\mathrm{c}}$ & 3.76 \\
\hline n-6 & $7.52^{b}$ & $11.87^{\mathrm{a}}$ & $6.76^{b}$ & $7.12^{b}$ & 3.19 \\
\hline Ratio of n-3 / n-6 & $0.73^{a}$ & $0.1 .09^{b}$ & $0.61^{\mathrm{a}}$ & $0.49^{\mathrm{a}}$ & 0.36 \\
\hline PUFA & $13.71^{\mathrm{a}}$ & $24.84^{b}$ & $10.72^{c}$ & $10.38^{c}$ & 6.30 \\
\hline SFA & $26.44^{\mathrm{a}}$ & $31.55^{\mathrm{b}}$ & $24.06^{\mathrm{C}}$ & $24.68^{c}$ & 8.85 \\
\hline PUFA / SFA & $0.57^{\mathrm{ab}}$ & $0.78^{a}$ & $0.44^{c}$ & $0.41^{\mathrm{C}}$ & 0.31 \\
\hline MUFA & $3.04^{\mathrm{a}}$ & $3.51^{b}$ & $1.85^{c}$ & $1.94^{\mathrm{C}}$ & 4.18 \\
\hline
\end{tabular}

a,b,c Values in each row that do not have any common letter are significantly ( $\mathrm{P} \leq 0.05)$ different.

\section{Discussion}

In the first Experiment, adding biological antioxidants, n-3 FA and $\alpha$-tocopherol after thawing, led to the higher percentage of progressively motile sperm. In a study conducted by Badr et al. (21) fortified extender with palmitic acid or linoleic acid (n-6) improved the post-thawed sperm motility and viability. Using the treated sperm for in vitro fertilization (IVF) led to better results in IVF and blastocyst formation $(22,23)$. In contrast, the results from a study on bulls conducted by Amorim et al. (24) revealed that extender supplementation with vitamin A, cod liver and flaxseed oil as a n-3 fatty acids source loaded on cyclodextrin could not improve sperm quality after thawing 
process.

The results of the present study showed that the levels of DHA, n-3 FA, the ratio of n-3 / n- 6 and PUFA in FA group were higher compared with the groups without FA. These findings revealed that embedment of n-3 FA into sperm cell membrane could have positive effects on sperm quality. Prior to freezing, DHA content was higher in FA group compare to group, which had no FA. However, this content was a dramatically decreased in both groups following the thawing process. These findings could be a result of enhanced lipid peroxidation, which is a consequence of spermatozoa cryopreservation (10). Also Maldjian et al. (12) stated that the decrease in the content of polyunsaturated fatty acids, could be a result of an increase in the amount of saturated fatty acids which taken up by sperm membrane or passively bound to the sperm membranes. Finally, all the mentioned mechanisms may lead to a decrease in the proportion of the LCPUFA. When n-3 FA applied to semen extender, DHA content of lipid composition in sperm membrane increased significantly. Thus, the valuable effects of n-3 FA on sperm quality is rationalized by considering the importance of DHA proportion in sperm membrane lipids (22). A result from a study in men indicated that spermatozoa from asthenozoospermic, oligozoospermic and oligoasthenozoospermi individuals had a lower docosahexaenoic acid content compared with normozoospermic men. On the other hand, there is a significant positive correlation between DHA and sperm motility, sperm concentration and normal sperm morphology (22).

PUFA content of sperm membrane (head and tail) may elevate in response to n-3 FA supplementation. On the other hand, the higher PUFA proportion in sperm membrane led to improved membrane fluidity and consequently sperm motility is developed. Furthermore, analysis of monkey sperm (head and tail), for fatty acids composition, revealed that 1.1 and $19.6 \%$ (respectively for head and tail) of total fatty acids in sperm are composed of DHA. This result is in agreement with previous findings demonstrated that $99 \%$ of DHA content of sperm is presented in the sperm tail. Plasma membrane fluidity, stability and permeability are the critical characteristics of sperm. The major components of sperm plasma membrane that may contribute to the sperm critical characteristics are the differences between lipid composition in sperm head and tail. Therefore, high DHA level in the sperm tail may be necessary since they improve sperm motility via increasing membrane fluidity in the sperm tail (25). In addition, freezing tolerance increases as a result of improved fluidity and flexibility. Therefore, it may prevent sperm membrane disintegration caused by ice crystal formation during the freezing process. It is widely accepted that PUFA served as a key factor in cell movements, lipid metabolism, and oocyte infusion by sperm. Kelso et al. (26) showed that in aged bull sperm count and motility were significantly decreased that are the conse- quences of reduction in sperm phospholipid DHA content (27). In a study conducted by Safarinejad et al. (28) the blood plasma PUFA content and spermatozoa PUFA composition were analyzed in two groups; men with idiopathic oligoasthenoteratozoospermia infertility and normal men. The results revealed that n-3 FA concentration was lower in plasma and spermatozoa of infertile men compare to the normal men (25).

There have been several studies with regards to the supplementation of fish oil into the rooster (10), boar (12) goat (29), sheep (30) and turkey (31) diet. The results revealed that the diet supplemented with fish oil led to an increase in sperm progressive motility. These reports were in agreement of the results of the present study.

Results of the present study revealed that the different concentration of $\alpha$-tocopherol supplemented with semen extender had the great impact on sperm progressive motility. In consistent with our experiment, findings from a study conducted by Jeong et al. (32) showed that fortified extender with $\alpha$-tocopherol led to more protection against oxidative damage. Thus, motility and progressive motility of sperm increased significantly. Previously, it was demonstrated that sperm morphogenesis led to a dramatic loose of internal deposition of antioxidant enzymes. Therefore, content of extracellular antioxidants have a pivotal role in prevention of oxidative damage caused by any kinds of stressors (31). Antioxidative system enables antioxidant agents to break the covalent bands that reactive oxygen species (ROS) form between fatty acid side chains in membrane lipids. This potential is prominent, especially in $\alpha$-tocopherol. This result showed that $\alpha$-tocopherol is a major component participating in membrane integrity and has a pivotal role in reducing membrane damage caused by excessive ROS production during cryopreservation. Maldjian et al. (12) showed that the application of egg yolks, to prepare semen extender, supplemented with n-3 fatty acids but any kinds of antioxidant had no significant effects on critical characteristics of sperm following cryopreservation. While results of the present study revealed that using FA concomitant with $\alpha$-tocopherol led to a significant increase in the quality of post-thawed sperm. Therefore, to gain the positive effects of n-3 FA supplementation in semen extender, using an antioxidant besides n-3 FA would be an obligation.

In conclusion, the present study showed that adding a source of n-3 FA and alfa-tocophrol to an extender can significantly improve the in vitro characteristics of ovine sperm. The highest and most suitable point of sperm quality after the freezing was observed in the highest level of fatty acid and vitamin E. It may be due to effective incorporation of PUFA especially DHA with the cell membrane before freezing and protection of cell membrane. Increased PMOT could be a result of DHA aggregation in the sperm flagellum area. Improved viability may be related to the increased membrane resistance to disinte- 
gration caused by ice crystal as a result of increased proportion of n-3 FA in plasma membrane.

\section{Acknowledgements}

There are no acknowledgments.

\section{Authors' Contribution}

Study design was conducted by A. Towhidi and S. Zeinoaldini. The experiment was performed by R. Ardebili and AH. Nasiri. Article written and prepared by A. Towhidi and N. Dadashpour Davachi.

\section{Financial Disclosure}

There is no conflict of interest.

\section{Funding / Support}

Present study is supported by University of Tehran, Department of Animal Science.

\section{References}

1. Watson PF. The causes of reduced fertility with cryopreserved semen. Anim Reproal sci. 2000;60:481-492.

2. Salamon S, Maxwell WMC. Storage of ram semen. Animal reproduction science. 2000;62(1):77-111.

3. Purdy PH. A review on goat sperm cryopreservation. Small Rumin Res. 2006;63(3):215-225.

4. Ansari M, Towhidi A, Moradi Shahrbabak M, Bahreini M. Docosahexaenoic acid and $\alpha$-tocopherol improve sperm cryosurvival in goat. SlovakJ Anim Sci. 2012;45(1):7-13.

5. Gil Jorge, Lundeheim Nils, Söderquist Lennart, RodríguezMartinez Heriberto. Influence of extender, temperature, and addition of glycerol on post-thaw sperm parameters in ram semen. Theriogenology. 2003;59(5):1241-1255.

6. Gil J, Lundeheim N, Soderquist L, Rodriguez-Martinez H. Influence of extender, temperature, and addition of glycerol on post-thaw sperm parameters in ram semen. Theriogenology. 2003;59(5-6):1241-55.

7. van Wagtendonk-de Leeuw AM, Haring RM, Kaal-Lansbergen LMTE, den Daas JHG. Fertility results using bovine semen cryopreserved with extenders based on egg yolk and soy bean extract. Theriogenology. 2000;54(1):57-67.

8. Bousseau S, Brillard JP, Marquant-Le Guienne B, Guérin B, Camus A, Lechat M. Comparison of bacteriological qualities of various egg yolk sources and the in vitro and in vivo fertilizing potential of bovine semen frozen in egg yolk or lecithin based diluents. Theriogenology. 1998;50(5):699-706.

9. Flesch Frits M, Gadella Barend M. Dynamics of the mammalian sperm plasma membrane in the process of fertilization. Biochimica et Biophysica Acta (BBA) - Reviews on Biomembranes. 2000;1469(3):197-235.

10. Parks John E, Lynch Daniel V. Lipid composition and thermotropic phase behavior of boar, bull, stallion, and rooster sperm membranes. Cryobiology. 1992;29(2):255-266.

11. Poulos A, Darin-Bennett Annabelle, White IG. The phospholipidbound fatty acids and aldehydes of mammalian spermatozoa. Comparative Biochemistry and Physiology Part B: Comparat Biochem. 1973;46(3):541-549.

12. Maldjian A, Pizzi F, Gliozzi T, Cerolini S, Penny P, Noble R. Changes in sperm quality and lipid composition during cryopreservation of boar semen. Theriogenology. 2005;63(2):411-21.

13. Rooke JA, Shao CC, Speake BK. Effects of feeding tuna oil on the lipid composition of pig spermatozoa and in vitro characteristics of semen. Reproduction. 2001;121(2):315-22.

14. Bwanga CO. Cryopreservation of boar semen. Acta Vet Scandinavica I. 1991;32:431-53.

15. Kelso KA, Cerolini S, Noble RC, Sparks NHC, Speake BK. Lipid and antioxidant changes in semen of broiler fowl from 25 to 60 weeks of age. Journal of Reproduction and Fertility. 1996;106(2):201-206.

16. Ijaz A, Hussain A, Aleem M, Yousaf MS, Rehman H. Butylated hydroxytoluene inclusion in semen extender improves the postthawed semen quality of Nili-Ravi buffalo (Bubalus bubalis). Theriogenology. 2009;71(8):1326-9.

17. Lin DS, Connor WE, Wolf DP, Neuringer M, Hachey DL. Unique lipids of primate spermatozoa: desmosterol and docosahexaenoic acid.J Lipid Res. 1993;34(3):491-9.

18. Sariozkan S, Bucak MN, Tuncer PB, Tasdemir U, Kinet H, Ulutas PA. Effects of different extenders and centrifugation / washing on postthaw microscopic-oxidative stress parameters and fertilizing ability of Angora buck sperm. Theriogenology. 2010;73(3):316-23.

19. Surai PF, Noble RC, Sparks NH, Speake BK. Effect of long-term supplementation with arachidonic or docosahexaenoic acids on sperm production in the broiler chicken. J Reprod Fertil. 2000;120(2):257-64.

20. Folch J, Lees M, Sloane Stanley GH. A simple method for the isolation and purification of total lipides from animal tissues. J Biol Chem. 1957;226(1):497-509.

21. Badr MR, Abdel-Malak MG, Shaker MH. Influence of some fatty acids and cholesterol addition to semen extender on freezability and in vitro fertilizing potentials of ram spermatozoa. Assiut Vet Med J. 2004;50(101):304-18.

22. Aksoy Y, Aksoy H, Altinkaynak K, Aydin HR, Ozkan A. Sperm fatty acid composition in subfertile men. Prostaglandins Leukot Essent Fatty Acids. 2006;75(2):75-9.

23. Zeinoaldini S, Jafari Z, Sarmast F, Torbati E, Dadashpour Davachi N. Different Harvesting Techniques Used in Ovine in vitro Embryo Production. Scimetr. 2013;1(1).

24. Amorim EAM, Graham JK, Spizziri B, Meyers M, Amorim LS, Torres CAA, editors. The effect of adding cholesterol, vitamin A, cod liver or flax oil loaded cyclodextrin on bull sperm cryosurvival.16th Int Cong Anim Reprod.; 2008. p. 29-P006.

25. Connor WE, Lin DS, Wolf DP, Alexander M. Uneven distribution of desmosterol and docosahexaenoic acid in the heads and tails of monkey sperm. J Lipid Res. 1998;39(7):1404-11.

26. Kelso KA, Redpath A, Noble RC, Speake BK. Lipid and antioxidant changes in spermatozoa and seminal plasma throughout the reproductive period of bulls. J Reprod Fertil. 1997;109(1):1-6.

27. Cerolini S, Zaniboni L, Maldjian A, Gliozzi T. Effect of docosahexaenoic acid and $\alpha$-tocopherol enrichment in chicken sperm on semen quality, sperm lipid composition and susceptibility to peroxidation. Theriogenology. 2006;66(4):877-86.

28. Safarinejad MR, Hosseini SY, Dadkhah F, Asgari MA. Relationship of omega-3 and omega- 6 fatty acids with semen characteristics, and anti-oxidant status of seminal plasma: a comparison between fertile and infertile men. Clin Nutr. 2010;29(1):100-5.

29. Dolatpanah MB, Towhidi A, Farshad A, Rshidi A, Rezayazdi K. Effect of dietary fish oil on semen quality of goats. Asian-Aust Anim Sci. 2008;21(1):29-34.

30. Samadian F, Towhidi A, Rezayazdi K, Bahreini M. Effects of dietary n-3 fatty acids on characteristics and lipid composition of ovine sperm. Animal. 2010;4(12):2017-22.

31. Zaniboni L, Rizzi R, Cerolini S. Combined effect of DHA and $\alpha$-tocopherol enrichment on sperm quality and fertility in the turkey. Theriogenology. 2006;65(9):1813-27.

32. Jeong YJ, Kim MK, Song HJ, Kang EJ, Ock SA, Kumar BM, et al. Effect of $\alpha$-tocopherol supplementation during boar semen cryopreservation on sperm characteristics and expression of apoptosis related genes. Cryobiology. 2009;58(2):181-9. 